\title{
Sub-cycle atomic-scale forces coherently control a single-molecule switch
}

https://doi.org/10.1038/s41586-020-2620-2

Received: 16 May 2019

Accepted: 30 June 2020

Published online: 2 September 2020

Check for updates

\author{
Dominik Peller', Lukas Z. Kastner', Thomas Buchner', Carmen Roelcke', Florian Albrecht ${ }^{1}{ }^{1}$, \\ Nikolaj Moll ${ }^{2}$, Rupert Huber ${ }^{1 凶}$ \& Jascha Repp ${ }^{1 凶}$
}

Scanning probe techniques can leverage atomically precise forces to sculpt matter at surfaces, atom by atom. These forces have been applied quasi-statically to create surface structures ${ }^{1-7}$ and influence chemical processes ${ }^{8,9}$, but exploiting local dynamics ${ }^{10-14}$ to realize coherent control on the atomic scale remains an intriguing prospect. Chemical reactions ${ }^{15-17}$, conformational changes ${ }^{18,19}$ and desorption ${ }^{20}$ have been followed on ultrafast timescales, but directly exerting femtosecond forces on individual atoms to selectively induce molecular motion has yet to be realized. Here we show that the near field of a terahertz wave confined to an atomically sharp tip provides femtosecond atomic-scale forces that selectively induce coherent hindered rotation in the molecular frame of a bistable magnesium phthalocyanine molecule. Combining lightwave-driven scanning tunnelling microscopy ${ }^{21-24}$ with ultrafast action spectroscopy ${ }^{10,13}$, we find that the induced rotation modulates the probability of the molecule switching between its two stable adsorption geometries by up to 39 per cent. Mapping the response of the molecule in space and time confirms that the force acts on the atomic scale and within less than an optical cycle (that is, faster than an oscillation period of the carrier wave of light). We anticipate that our strategy might ultimately enable the coherent manipulation of individual atoms within single molecules or solids so that chemical reactions and ultrafast phase transitions can be manipulated on their intrinsic spatio-temporal scales.
A magnesium phthalocyanine (MgPc) molecule adsorbed on the chlorine sites of a sodium chloride $(\mathrm{NaCl})$ thin film constitutes a single-molecule switch, with the axes of the neutral molecule rotated by an azimuthal angle of either $\phi_{1}=+10^{\circ}$ or $\phi_{\mathrm{r}}=-10^{\circ}$ with respect to those of the underlying surface (Fig. 1a, b). This defines two stable structural configurations, denoted $|l\rangle$ and $|r\rangle$, with the adsorption energy $U(\phi)$ a double-well potential with degenerate minima at $\phi_{1}$ and $\phi_{\mathrm{r}}$ (Fig. 1b) $)^{25,26}$. The most direct initiation of atomic motion in this switch is to exert a mechanical force on the molecular frame, leaving the system in its electronic ground state. Although static forces available with scanning probe microscopy ${ }^{1-4,7}$ could induce a static excursion, a time-dependent ultrafast force pulse, $\mathbf{F}(t)$, may prepare a coherent frustrated rotation of the molecule (Fig. 1c, d) about one of the potential minima. We will see below that such an atomically sharp ultrafast force can indeed be provided by the oscillating carrier wave of a strong terahertz pulse coupled to the metallic tip of a scanning tunnelling microscope (STM). The force-induced all-structural wavepacket dynamics can be strong enough even to influence the probability of the molecule switching between its stable states.

Our idea is to interrogate this switching probability with an ultrafast time-delayed electron injection event, available with a femtosecond lightwave-driven STM ${ }^{21-24}$. Thereby, the carrier wave of a laser pulse focused onto the STM junction serves as a transient bias voltage, setting a femtosecond time window (here around $100 \mathrm{fs}$ ) for the injection of one single electron ${ }^{22}$ into the lowest unoccupied molecular orbital (LUMO) of an individual MgPc molecule. Because the charged molecule is subject to a modified potential $U^{*}(\phi)$ whose minimum lies around $\phi=0^{\circ}$ (Fig. 1f), electron injection prompts the molecule to rotate transiently towards $\phi=0^{\circ}$ (refs. ${ }^{25,26}$ ). When the additional electron has tunnelled into the substrate after a short lifetime $\tau_{\text {charge, }}$, the double-well potential $U(\phi)$ is restored and the molecule relaxes towards either $|l\rangle$ or $|r\rangle$ (Fig. 1e). Only resonant tunnelling into or out of one of the molecular orbitals results in temporary charging and associated actuation of the molecular switch, whereas tunnelling at biases well below any orbital resonance does not, as confirmed in steady-state STM experiments (see Methods).

To assess the impact of an ultrafast atomic-scale force on the single-molecule switch, it is important first to understand the switching behaviour triggered by lightwave-induced resonant electron injection, in the absence of a force pulse $\mathbf{F}(t)$. In contrast to the recent demonstration of lightwave-driven $\mathrm{STM}^{21,22}$, we track not the terahertz-induced tunnelling current but the structural change of the single-molecule switch. Since the system does not relax into the same initial state after every laser shot, but rather toggles between the two stable states, the outcome of every laser shot needs to be evaluated, one by one. We extend such single-molecule action spectroscopy ${ }^{19}$ from combined sub-ångström spatial and ultrafast temporal precision ${ }^{10,13}$ to a regime 

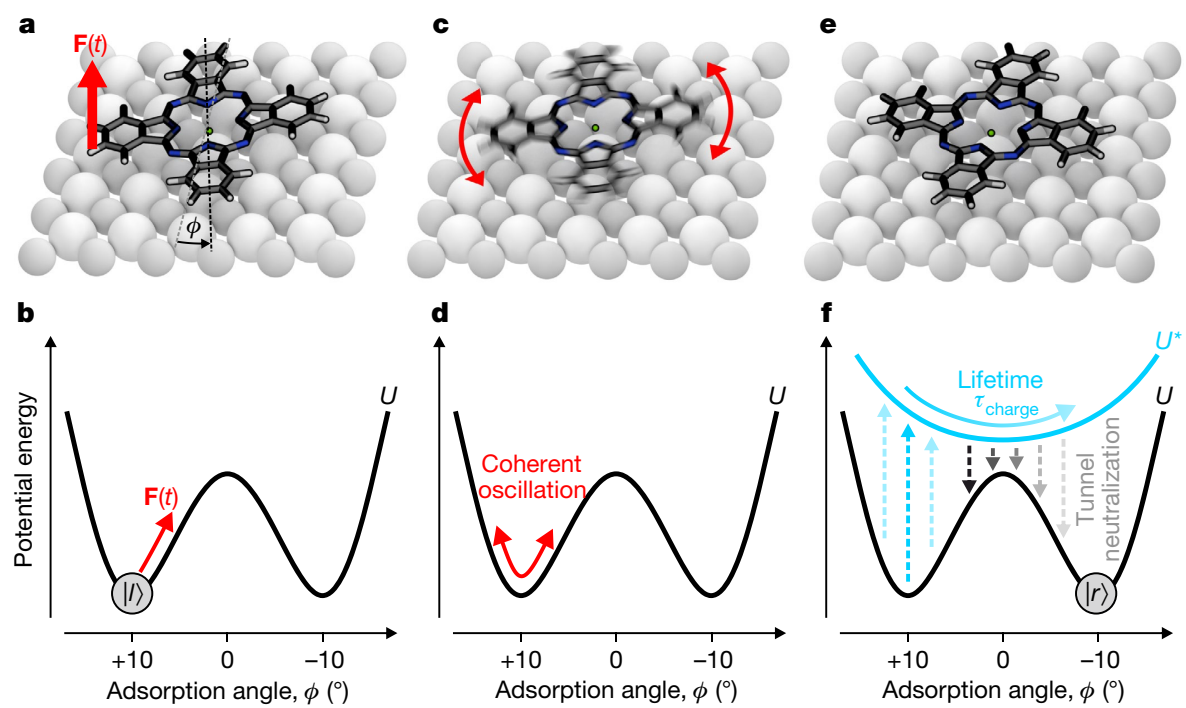

Fig. 1 | Coherent control of structural dynamics of a single-molecule switch by a local ultrafast force stimulus. a, In equilibrium, the neutral MgPc molecule adsorbs in either of two degenerate, mirror-symmetric ground states $|l\rangle$ (geometry shown) and $|r\rangle$, where the molecule's axes are rotated by $\phi_{1}=+10^{\circ}$ and $\phi_{\mathrm{r}}=-10^{\circ}$ with respect to those of the underlying $\mathrm{NaCl}$ lattice, respectively. b. The associated potential energy landscape features two minima separated by a barrier. $\mathbf{c}-\mathbf{e}$, Applying an atomically local force stimulus $\mathbf{F}(t)$ might efficiently and coherently drive an in-plane rotational oscillation of the molecule (c, d), which could be exploited to coherently control transient

in which the oscillating carrier wave itself controls dynamics on timescales shorter than a cycle of light.

The terahertz laser source inducing femtosecond electron injection is operated in single-shot mode to toggle the single molecule switch with a certain probability. Steady-state imaging at low bias voltages monitors the adsorption geometries $|l\rangle$ and $|r\rangle$ without switching between them (Fig. 2a, b; see Methods for details). When the tip is
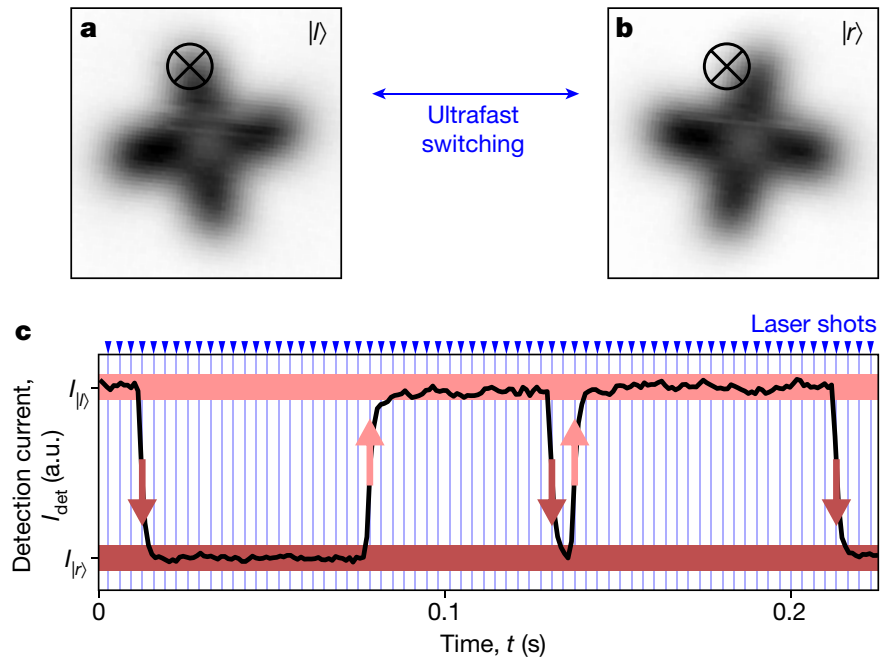

Fig. 2 | Path-selective map of the single-pulse switching probability. a, b, Low-voltage maps of the tunnelling current at constant height display the in-plane rotation of the neutral molecule in both adsorption geometries separately (bias voltage, $6 \mathrm{mV}$; image size, $20 \AA \times 20 \AA$; linear grey scale, $0-2 \mathrm{pA}$ ). c, Keeping the tip at a fixed position above the molecule (for example, at the crossed circles in $\mathbf{a}$ and $\mathbf{b}$ ), the orientation of the molecule can be monitored by a non-resonant detection current $I_{\text {det }}$ (black) while laser shots (blue vertical lines) repeatedly inject single electrons into the LUMO, statistically triggering switching back and forth between the adsorption geometries (arrows). Every switching event manifests as an abrupt change of the detection current, which switching dynamics into state $|r\rangle$ (e).f, Because the charged molecule energetically prefers aligning with the lattice directions, ultrafast singleelectron injection in a lightwave STM (vertical transition) establishes a new energy landscape (blue curve). This sudden change triggers an in-plane rotation of the molecule towards $\phi=0^{\circ}$, strongly influenced by previously induced rotational dynamics. Tunnel neutralization can stochastically occur at different instants in time (decay lifetime $\tau_{\text {charge; }}$; multiple downward arrows), such that the outcome of every individual switching process is governed by quantum probability.

positioned above the molecule in such a way that the tunnelling current differs for $|l\rangle$ and $|r\rangle$ (crossed circles in Fig. 2a, b), we can use this detection current, $I_{\mathrm{det}}$, to monitor the adsorption geometry continuously even without taking images. Figure $2 c$ displays a typical time trace of $I_{\mathrm{det}}$ acquired over $0.23 \mathrm{~s}$ while individual terahertz pulses repeatedly injected single electrons into the LUMO. Whenever an electron injection triggers the molecule to switch its adsorption geometry, the
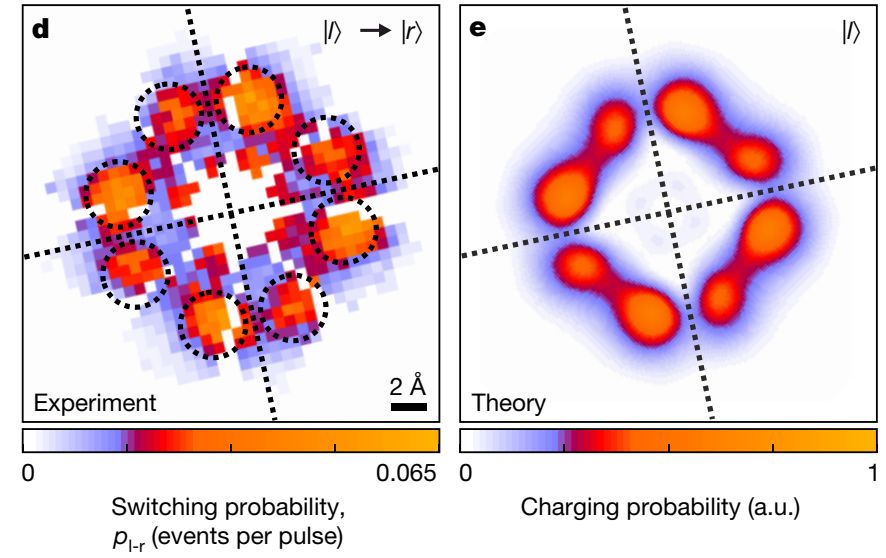

is registered for each laser shot, separately for both directions. Systematically recording the switching statistics on different positions across the molecule, we obtain spatial maps of the switching probabilities for both directions,

$p_{\text {l-r }}$ from $|l\rangle$ to $|r\rangle(\mathbf{d})$ and $p_{\mathrm{r}-1}$ from $|r\rangle$ to $|l\rangle$ (Extended Data Fig. 2), which reveal rich sub-molecular features and a marked direction dependence. Pixels where switching cannot be detected are depicted in white. Eight areas of enhanced switching probability stand out (dashed circles), four of which are more prominent. The axes of the molecule in the initial state are indicated by dashed lines. e, Simulated charging probability by tunnelling into a LUMO in state $|l\rangle$ in arbitrary units (a.u.). 

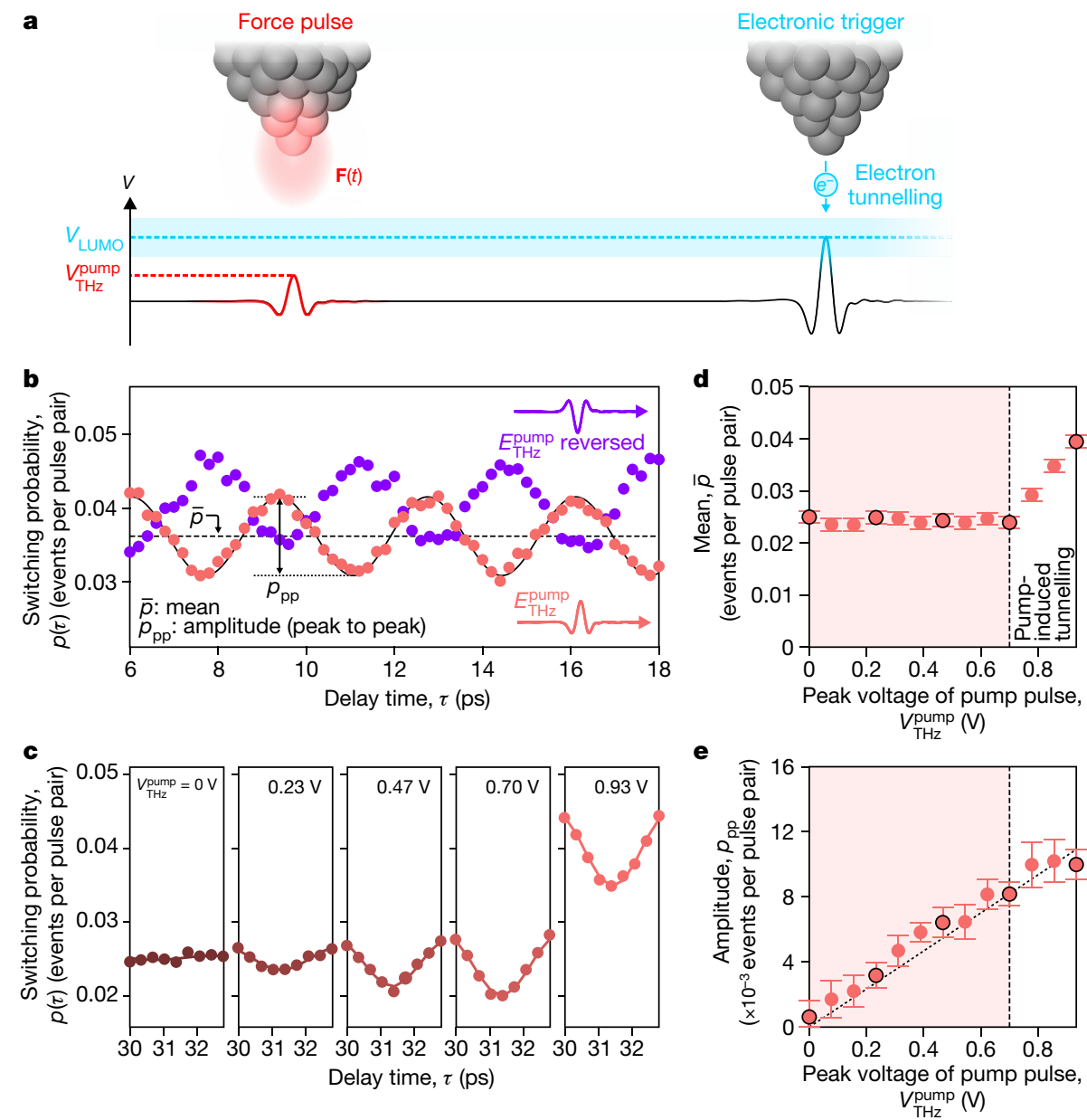

Fig. 3 | Ultrafast force stimuli coherently control the single-molecule switching probability. a, In a pump-probe scheme, pairs of terahertz pulses with an adjustable delay time $\tau$ induce switching events. The pump pulses are tuned below the threshold of electron tunnelling, whereas the probe pulses serve to inject single electrons and interrogate molecular dynamics via time-delayed switching events. b. The switching probability as a function of delay time exhibits a marked oscillation at a frequency of $0.3 \mathrm{THz}$ (red data points, $V_{\mathrm{THz}}^{\text {pump }}=0.7 \mathrm{~V}$ ). A reversal of the polarity of the pump pulse coherently inverts the phase of the oscillatory signal (violet data points).c, The mechanism by which the pump pulse acts on the molecule is examined by recording one oscillation period of the time-resolved switching probability traces, $p(\tau)$, while systematically varying the pump pulse's field strength (additional traces shown in Extended Data Fig. 4). The latter is indicated in the top right corner of each panel, expressed as the peak voltage of the corresponding transient current changes sharply from $I_{|l\rangle}$ to $I_{|r\rangle}$ or vice versa, allowing us to detect every switching event shot by shot, with excellent fidelity. All events are registered for both directions separately (arrows in Fig. 2c), such that the probability per light pulse $p_{1-\mathrm{r}}$ to switch from $|l\rangle$ to $|r\rangle$ and the probability $p_{\mathrm{r}-1}$ for the reverse direction can be distinguished.

Both $p_{\mathrm{l}-\mathrm{r}}$ and $p_{\mathrm{r}-\mathrm{l}}$ depend on the precise lateral STM tip position $(x, y)$ when injecting an electron into the molecule. From thousands of terahertz-induced switching time traces we obtain separate maps of local probabilities $p_{\mathrm{l}-\mathrm{r}}(x, y)$ (Fig. 2d) and $p_{\mathrm{r}-\mathrm{l}}(x, y)$ (Extended Data Fig. 2), which reveal rich sub-molecular features and a clear direction dependence. The $p_{1-r}(x, y)$ map closely resembles the calculated efficiency of local electron injection into the LUMO density of the initial-state configuration $|l\rangle$ (Fig. 2e; see Methods for details). Both maps feature eight outer lobes and nodal planes along the isoindole units (dashed lines), which reflect the in-plane orientation of the molecule. Moreover, four of the eight lobes are more prominent, which stems from an internal (calibrated via Extended Data Fig. 3). For each curve, the time-averaged mean, $\bar{p}$, and the modulation amplitude, $p_{\mathrm{pp}}$, are extracted by fitting a sine (solid lines in c) to the data.d, The mean as a function of field strength is constant up to $V_{\mathrm{THz}}^{\text {pump }}=0.7 \mathrm{~V}$ (vertical line) and then rises steeply. For $V_{\mathrm{THz}}^{\text {pump }}<0.7 \mathrm{~V}$, the pump pulse is too weak to inject electrons and only the probe pulse induces switching events. Consequently, the mean is constant irrespective of the pump field strength. Above this threshold, the pump pulse triggers additional electron tunnelling and contributes to the overall switching probability.e, By contrast, the modulation amplitude, $p_{\mathrm{pp}}$, scales linearly with the pump field strength throughout the measured range, indicating that it is the electric field of the pump pulse that leads to the coherent excitation. This picture is corroborated by the observed polarity dependence (b). No change in slope is observed at the critical value of $V_{\mathrm{THz}}^{\mathrm{pump}}=0.7 \mathrm{~V}$ (vertical line). Error bars in $\mathbf{d}$ and $\mathrm{e}$ are the standard deviations of $\bar{p}$ and $p_{\mathrm{pp}}$.

torsion of the molecule as revealed by density functional theory (DFT) calculations. The agreement between the calculated LUMO density map and the measured switching probability map indicates that the switching probability scales linearly with the electron injection efficiency; in other words, the probability that an injected electron triggers a switching event is position-independent. By relating the lightwave-induced current to the switching rate, this probability is estimated to be $21 \% \pm 3 \%$. If the amplitude of the terahertz pulse is lowered by, for example, $25 \%$, such that the pulse cannot induce tunnelling into the LUMO, the switching probability drops to zero.

To demonstrate that the switching probability of the single MgPc molecule can be influenced by an ultrafast force pulse that induces a coherent, predominantly rotational motion of the molecular frame, we next perform a pump-probe experiment. The pump acts as ultrafast force pulse, and a time-delayed probe injects an electron into the LUMO to induce single-molecule switching (Fig. 3a), which is evaluated for 
a
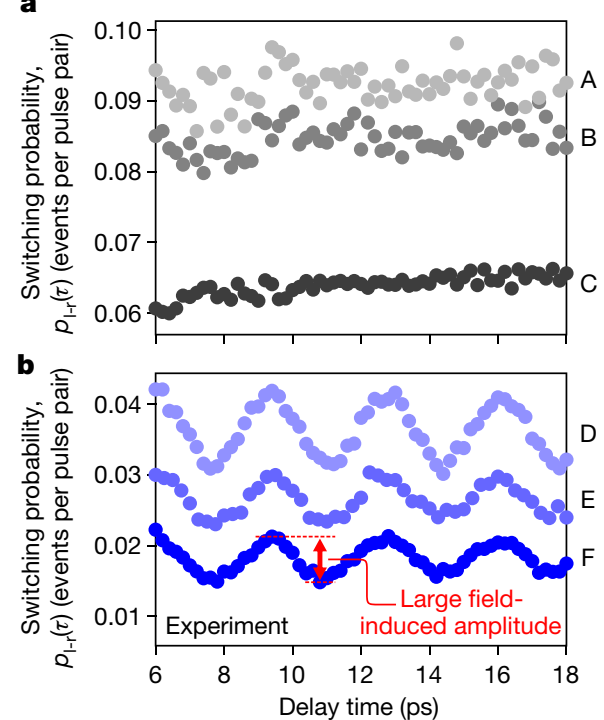

Fig. 4 | Atomic spatial selectivity of the femtosecond force stimulus. a, b, Six traces of the time-resolved switching probability at different tip positions (indicated by crosses labelled $A$ to $F$ in $\mathbf{c}$ ) reveal strong lateral variations in the amplitude of the force-induced modulation.c, The colour map illustrates how a local out-of-plane electric field centred at different positions (pixel coordinates) acts as an atomic-scale force and induces an in-plane rotation of the MgPc molecule of different amplitudes (colour bar), as computed by DFT calculations (see Methods for details). A top view of the calculated geometry of the molecule without applied field is overlaid with selected hydrogen atoms highlighted in pink. d, Top view of this molecular geometry including the underlying $\mathrm{NaCl}$ lattice. e, Schematic side view of the adsorption geometry, in which the $z$ excursion of the calculated atomic positions in MgPc is exaggerated by a factor of 6 . The geometry suggests that the interaction of specific hydrogen atoms (pink) with the underlying chlorine atoms (intense green) and sodium atoms (intense blue) dominates the energetics concerning the azimuthal orientation (see also Extended Data Fig. 6). This is consistent with the observation in theory and experiment that local fields applying forces close to these atoms induce the strongest molecular rotation and coherent dynamics. every laser shot to retrieve the switching probability $p(\tau)$ as a function of the pump-probe delay time $\tau$. We implement the ultrafast force by exploiting the oscillating near field of a terahertz pulse localized at the metallic tunnelling tip, with the near-field amplitude fixed at a value below that required to inject an electron into the molecule. This ensures that, in contrast to a recent measurement of single-molecule vertical vibration ${ }^{22}$, structural dynamics cannot be a result of temporary charging. As shown in Fig. 3b, the pump pulse nevertheless imprints a marked oscillation with a $0.3-\mathrm{THz}$ frequency on the switching probability $p(\tau)$. The oscillation frequency matches the frustrated in-plane rotation frequency calculated by DFT. Given that the switching trajectory is predominantly such a rotation of the molecule, this agreement in frequency suggests that the pump pulse triggers this motion. As will be corroborated below, this interaction is purely mediated by the electric near field acting as an atomically localized force transient.

To confirm this interpretation, we vary the pump field strength and hence the transient voltage applied across the tunnelling junction, $V_{\mathrm{THz}}^{\text {pump }}$, from below to above the threshold for electron injection into the LUMO (seeExtended Data Fig. 3 for the field-to-voltage calibration), while keeping the amplitude of the probe pulse constant. Five typical $p(\tau)$ traces (see Extended Data Fig. 4 for more traces) are shown in Fig. 3c, revealing that the pump pulse induces a marked modulation of $p(\tau)$ and adds a delay-independent positive offset. These two effects scale markedly differently as a function of $V_{\mathrm{THz}}^{\text {pump }}$ (Fig. 3d, e). The mean of the switching probability, $\bar{p}$, (Fig. 3d) remains flat for weak pump fields but increases steeply once $V_{\mathrm{THz}}^{\text {pump }}$ exceeds the voltage required to induce electron tunnelling into the LUMO. We can thus unequivocally attribute the steep increase of $\bar{p}$ to switching events triggered by electron tunnelling induced by the pump pulse. Conversely, for weak pump fields only the probe pulse triggers electron tunnelling, which leads to the constant mean of $\bar{p} \approx 0.025$. In sharp contrast, the amplitude of the coherent modulation in the switching probability, $p_{\mathrm{pp}}$, (Fig. 3e) increases linearly with the pump field, starting from zero, over the entire field range. Even pump pulses that are too weak to induce electron tunnelling into the LUMO strongly influence the switching probability of the probe pulse. This indicates that the electric field of the pump pulse itself coherently excites the system, which is further corroborated by the fact that reversing the polarity of the pump field leads to an oscillation of $p(\tau)$ at the same frequency but with opposite phase (see Fig. 3b). These results demonstrate that while local electron injection is required to trigger a switching event, pump pulses below the tunnelling threshold can induce coherent motion in the system by virtue of an ultrafast electric-field stimulus. The electric field directly exerts forces on those atoms that carry a net charge, while the field's inhomogeneity results in forces via the polarizability of each atom. Hence, irrespective of the mechanism, the electric field of the pulse translates into an ultrafast force transient acting locally.

Pump-probe experiments at varying tip positions while keeping the pump field below the tunnelling threshold show that both the mean switching rate, $\bar{p}$, and the modulation amplitude, $p_{\mathrm{pp}}$, depend critically on the location over the molecule, yet their spatial patterns are distinctly different. We find local regions where-less than $5 \AA$ apart-the force-induced modulation, $p_{\mathrm{pp}}$, is either absent (Fig. 4a) or maximized (Fig. 4b), verifying that the field acts locally, confined to the Ångström scale. Conversely, $\bar{p}$ is determined by the mean efficiency of the probe pulse triggering a switching event, following the LUMO tunnelling probability as in Fig. 2 . We observe regions where $\bar{p}$ is large but $p_{\mathrm{pp}}$ is absent (Fig. 4a) and others where $\bar{p}$ is moderate but $p_{\mathrm{pp}}$ is maximized (Fig. 4b), confirming that the local force acts independently of electronic excitation. We provide a visualization of these observations in Supplementary Video 1, which maps out the spatio-temporally resolved switching statistics across a single molecule. The coherent control demonstrated here enables modulation amplitudes of up to $39 \%$ (Extended Data Fig. 5).

The electric terahertz near field in the STM junction is expected to be oriented predominantly perpendicularly to the surface, because the metal substrate efficiently screens any in-plane component. At first glance, it may come as a surprise that this field can efficiently modulate 
a switching process that requires mainly an in-plane rotation. Yet a microscopic model convincingly corroborates this scenario. We performed DFT calculations, in which the geometry of the combined system of molecule and $\mathrm{NaCl}$ layer is relaxed. Figure 4c shows a top view of the molecule in state $|l\rangle$ with no field applied. Upon inclusion of a local out-of-plane electric field to model the effect of the pump pulse onto the molecule's azimuthal orientation we observe the molecule undergoing a small azimuthal excursion that depends sensitively on the precise position of the local field (see colour scale in Fig. 4c). The pattern of the local efficiency of a rotational excitation can be rationalized as follows. The atomic positions of the relaxed geometry (Fig. 4d, e) suggest that the interaction of specific hydrogen atoms (highlighted in pink) with the underlying chlorine (highlighted in green) and sodium atoms (highlighted in blue) dominate the azimuthal energetics, giving rise to the double-well energy landscape (see Extended Data Fig. 6). Figure 4c strikingly illustrates that, according to the calculation, local fields applied at these atoms induce an appreciable rotation, whereas fields applied elsewhere do not. These rotations are too small to directly induce switching of the orientation, consistent with the experimental observation that without electron injection no switching is observed. However, they are large enough to trigger an in-plane frustrated-rotational motion that will modulate the switching probability of a later probe pulse and thereby allow for coherent control. The calculated pattern of the local efficiency of a rotational excitation agrees very well with the experimentally observed variations of the modulation amplitude of $p(\tau)$ (Fig. 4a, b).

The ultrafast coherent atomic manipulation demonstrated here paves the way for path-selective real-space reaction microscopy with sub-cycle and atomic precision. Ultrafast structural transitions of a variety of quantum systems-ranging from single molecules to solids-can now be controlled on their natural time and length scales. We anticipate that combining our control scheme with recent probing of ultrafast forces ${ }^{27,28}$ may pave the way to sub-molecular lightwave force microscopy. In the near future, we envisage resolving and manipulating the dynamics of chemical reactions, phase transitions in strongly correlated materials ${ }^{29}$ and the quantum properties of biomolecular systems ${ }^{30}$.

\section{Online content}

Any methods, additional references, Nature Research reporting summaries, source data, extended data, supplementary information, acknowledgements, peer review information; details of author contributions and competing interests; and statements of data and code availability are available at https://doi.org/10.1038/s41586-020-2620-2.

1. Hla, S.-W., Bartels, L., Meyer, G. \& Rieder, K.-H. Inducing all steps of a chemical reaction with the scanning tunneling microscope tip: towards single molecule engineering. Phys. Rev. Lett. 85, 2777-2780 (2000)

2. Heinrich, A. J., Lutz, C. P., Gupta, J. A. \& Eigler, D. M. Molecule cascades. Science 298, 1381-1387 (2002).
3. Stroscio, J. A. \& Celotta, R. J. Controlling the dynamics of a single atom in lateral atom manipulation. Science 306, 242-247 (2004)

4. Ternes, M., Lutz, C. P., Hirjibehedin, C. F., Giessibl, F. J. \& Heinrich, A. J. The force needed to move an atom on a surface. Science 319, 1066-1069 (2008).

5. Pavliček, N. et al. Synthesis and characterization of triangulene. Nat. Nanotechnol. 12 308-311 (2017).

6. García-López, V. et al. Molecular machines open cell membranes. Nature 548, 567-572 (2017).

7. Esat, T., Friedrich, N., Tautz, F. S. \& Temirov, R. A standing molecule as a single-electron field emitter. Nature 558, 573-576 (2018).

8. Garcia-Manyes, S. \& Beedle, A. E. M. Steering chemical reactions with force. Nat. Rev. Chem. 1, 0083 (2017).

9. Ladenthin, J. N. et al. Force-induced tautomerization in a single molecule. Nat. Chem. 8 , 935-940 (2016).

10. Wu, S. \& Ho, W. Two-photon-induced hot-electron transfer to a single molecule in a scanning tunneling microscope. Phys. Rev. B 82, 085444 (2010).

11. Lee, J., Perdue, S. M., Rodriguez Perez, A. \& Apkarian, V. A. Vibronic motion with joint angstrom-femtosecond resolution observed through Fano progressions recorded within one molecule. ACS Nano 8, 54-63 (2014).

12. Yampolsky, S. et al. Seeing a single molecule vibrate through time-resolved coherent anti-Stokes Raman scattering. Nat. Photon. 8, 650-656 (2014)

13. Li, S., Chen, S., Li, J., Wu, R. \& Ho, W. Joint space-time coherent vibration driven conformational transitions in a single molecule. Phys. Rev. Lett. 119, 176002 (2017).

14. Lee, J., Crampton, K. T., Tallarida, N. \& Apkarian, V. A. Visualizing vibrational normal modes of a single molecule with atomically confined light. Nature 568, 78-82 (2019).

15. Wörner, H. J., Bertrand, J. B., Kartashov, D. V., Corkum, P. B. \& Villeneuve, D. M. Following a chemical reaction using high-harmonic interferometry. Nature 466, 604-607 (2010).

16. Wolter, B. et al. Ultrafast electron diffraction imaging of bond breaking in di-ionized acetylene. Science 354, 308-312 (2016).

17. Elsaesser, T. Introduction: ultrafast processes in chemistry. Chem. Rev. 117, 10621-10622 (2017).

18. Prokhorenko, V. I. et al. Coherent control of retinal isomerization in bacteriorhodopsin Science 313, 1257-1261 (2006)

19. Böckmann, H. et al. Near-field spectral response of optically excited scanning tunneling microscope junctions probed by single-molecule action spectroscopy. J. Phys. Chem. Lett. 10, 2068-2074 (2019).

20. Dürr, M., Biedermann, A., Hu, Z., Höfer, U. \& Heinz, T. F. Probing high-barrier pathways of surface reactions by scanning tunneling microscopy. Science 296, 1838-1841 (2002).

21. Cocker, T. L. et al. An ultrafast terahertz scanning tunnelling microscope. Nat. Photon. 7, 620-625 (2013)

22. Cocker, T. L., Peller, D., Yu, P., Repp, J. \& Huber, R. Tracking the ultrafast motion of a single molecule by femtosecond orbital imaging. Nature 539, 263-267 (2016).

23. Yoshioka, K. et al. Real-space coherent manipulation of electrons in a single tunnel junction by single-cycle terahertz electric fields. Nat. Photon. 10, 762-765 (2016).

24. Jelic, V. et al. Ultrafast terahertz control of extreme tunnel currents through single atoms on a silicon surface. Nat. Phys. 13, 591-598 (2017)

25. Miwa, K., Imada, H., Kawahara, S. \& Kim, Y. Effects of molecule-insulator interaction on geometric property of a single phthalocyanine molecule adsorbed on an ultrathin $\mathrm{NaCl}$ film. Phys. Rev. B 93, 165419 (2016).

26. Patera, L. L., Queck, F., Scheuerer, P., Moll, N. \& Repp, J. Accessing a charged intermediate state involved in the excitation of single molecules. Phys. Rev. Lett. 123 016001 (2019).

27. Jahng, J. et al. Ultrafast pump-probe force microscopy with nanoscale resolution Appl. Phys. Lett. 106, 083113 (2015)

28. Schumacher, Z., Spielhofer, A., Miyahara, Y. \& Grutter, P. The limit of time resolution in frequency modulation atomic force microscopy by a pump-probe approach. Appl. Phys. Lett. 110, 053111 (2017).

29. Eichberger, M. et al. Snapshots of cooperative atomic motions in the optical suppression of charge density waves. Nature 468, 799-802 (2010).

30. Scholes, G. D. et al. Using coherence to enhance function in chemical and biophysical systems. Nature 543, 647-656 (2017)

Publisher's note Springer Nature remains neutral with regard to jurisdictional claims in published maps and institutional affiliations.

(c) The Author(s), under exclusive licence to Springer Nature Limited 2020 


\section{Methods}

\section{STM setup}

The custom-built STM ${ }^{22}$ is based on a Besocke design and operates in ultrahigh vacuum (pressure about $7 \times 10^{-11} \mathrm{mbar}$ ) and at low temperatures down to $7 \mathrm{~K}$. The bias voltage is applied to the sample. A custom-built, low-temperature (around $100 \mathrm{~K})$, high-gain $\left(G=2.5 \times 10^{10} \mathrm{VA}^{-1}\right)$ preamplifier ( $I-V$ converter) is mounted in close proximity to the STM head. Terahertz radiation enters the vacuum chamber through a sapphire viewport as a collimated beam and is focused onto the tip by a parabolic mirror that is fixed to the STM scan unit.

\section{Sample preparation}

Sodium chloride is evaporated thermally onto a clean copper (111) sample under ultrahigh-vacuum conditions at about $275 \mathrm{~K}$ to form bilayers and trilayers. Magnesium phthalocyanine molecules are deposited onto the cold substrate $(T<15 \mathrm{~K})$ inside the STM scanner.

\section{Terahertz optical setup}

Intense, phase-locked terahertz pulses with a centre frequency of 1.1 THz are generated by tilted-pulse-front optical rectification of femtosecond near-infrared pulses (centre wavelength, $1,028 \mathrm{~nm}$; pulse duration full width at half-maximum, $250 \mathrm{fs}$ ) from a regenerative ytterbium-doped potassium gadolinium tungstate (Yb:KGW) laser amplifier (repetition rate tuneable from $0.61 \mathrm{MHz}$ down to single shot) in lithium niobate. Pairs of mutually delayed terahertz transients are prepared by transmitting the terahertz pulses through a Michelson interferometer, in which the computer-controlled position of one end mirror sets the delay time $\tau$. Using crossed wire-grid polarizers, the terahertz field amplitude is continuously controlled, for both pulses individually, without changing the waveform. Their field directions can be reversed separately.

\section{Time-integrated orbital imaging}

The statistical switching behaviour of the MgPc molecule upon charging is confirmed in a time-integrated way with conventional steady-state STM. When increasing the bias voltage such that electron tunnelling into the lowest unoccupied molecular orbital (LUMO) charges the molecule, the switch starts to toggle. In steady-state STM imaging of select orbitals, millions of sequential electron tunnelling events through these states are necessary to record a single pixel, resulting in countless switching events. The repeated toggling of the orientation results in a superposition of LUMO images for $|l\rangle$ and $|r\rangle$ and prevents a separate observation of the individual states (see Extended Data Fig. 1). Nonetheless, the ground-state geometry of the molecular frame can be determined by recording steady-state STM images at a bias voltage well below any molecular resonance, such that electrons must tunnel from the tip through the molecule into the substrate in a single step, without being able to transiently charge the molecule (Fig. 2a, b).

\section{Single-shot switching detection}

To resolve every single switching event ${ }^{31,32}$ of the molecule, a detection mechanism that allows us to continuously monitor the molecule's adsorption geometry is used, extending previously reported implementations of action spectroscopy in $\mathrm{STM}^{19,33-35}$. To this end, the tip is first positioned above the bare $\mathrm{NaCl}$ substrate close to the molecule that is to be examined. The tip height is determined by the feedback loop operating at a current setpoint of $2 \mathrm{pA}$ at a bias voltage of $20 \mathrm{mV}$ (bilayer $\mathrm{NaCl}$; data shown in Fig. 2 and Extended Data Fig. 2) or $220 \mathrm{mV}$ (trilayer $\mathrm{NaCl}$, all other data shown), respectively. Although we do not observe qualitative differences for the data acquired on bilayer and trilayer $\mathrm{NaCl}$, for consistency, all time-resolved data were recorded on a trilayer of $\mathrm{NaCl}$ only. After switching off the feedback loop, the tip is moved laterally at constant height to the desired measurement position above the molecule, where it remains throughout the measurement. Applying a constant bias voltage with typical values around $15 \mathrm{mV}$ (bilayer $\mathrm{NaCl}$ ) or $150 \mathrm{mV}$ (trilayer $\mathrm{NaCl}$ ) well below any of the molecular resonances drives a detection current $I_{\mathrm{det}}$ of a few picoamperes. Because this current is not accompanied by a transient charging of the molecule it does not result in switching events, yet it differs for both adsorption states.

Simultaneously, terahertz pulses or pairs of pulses with individually tuneable field strengths are coupled into the STM. For every set of measurements, the field strengths of the pulses are calibrated via an onset curve as shown in Extended Data Fig. 3, allowing us to control the voltage transiently applied across the tunnelling junction. When we choose the field strength of a terahertz pulse such that the voltage transient accesses the LUMO resonance with its apex, we controllably set a time window with a width of about $100 \mathrm{fs}$ for the injection of a single electron (ref. ${ }^{22}$ ). In the experiments presented, all lightwave-induced tunnelling rates remained below 0.4 electrons per pulse. With a certain probability, tunnelling injection leads to a switching event between the two adsorption geometries, resulting in a persistent change of the detection current. We continuously record a time trace of this detection current while repeatedly applying terahertz pulses, and all switching events manifest as abrupt changes of $I_{\text {det }}$. Observing these abrupt changes of $I_{\mathrm{det}}$ reveals every single switching event separately for both directions. The bandwidth of the STM current preamplifier leads to the finite rise time in the observed changes of $I_{\text {det }}$ (see Fig. 2c). To facilitate the separate detection of every event, the repetition rate of the laser pulse trains must therefore be adapted to the bandwidth of the STM current preamplifier.

To resolve the switching statistics with high precision, these experiments are repeated numerous times to observe a sufficient number of events for every parameter combination (fixed position, delay time and field strengths). In Fig. 3b, for example, every data point represents statistics extracted from a typical time trace where a total of 249,770 terahertz pulse pairs were coupled to the STM, $n \approx 159,000$ of which were incident with the molecule in one of the two orientations. This sequence of pulses triggers about $n \times p=5,760$ switching events observed in one switching direction, with a switching probability $p$. Statistically, every laser shot represents a Bernoulli trial and every sequence follows a binomial distribution. This yields a shot-noise limited signal-to-noise ratio of $\sqrt{n p}=76$, corresponding to a relative statistical uncertainty of $1.3 \%$. By fitting the data in Fig. 3b to a sine curve, we obtain an experimental relative deviation of $1.6 \%$ (standard deviation divided by mean).

\section{DFT calculations}

We performed DFT calculations of MgPc on a cluster of $\mathrm{NaCl}(100)$ using the FHI-aims code with numerical atomic orbitals as the basis functions ${ }^{36}$. The Perdew-Burke-Ernzerhof ${ }^{37}$ exchange-correlation functional with an admixture of exact exchange of 0.8 and a van der Waals method ${ }^{38,39}$ was applied for all calculations. As in the experiment, the molecule was centred on top of $\mathrm{CCl}^{-}$site. To identify the mechanisms that dominate the adsorption energetics with respect to the azimuthal orientation (Extended Data Fig. 6), geometry optimization was performed with the azimuthal angle being constrained to values in the range $0^{\circ}$ to $20^{\circ}$ by fixing one of the in-plane coordinates of two nitrogen atoms and relaxing all other degrees of freedom. The response of the coupled system of neutral molecule and $\mathrm{NaCl}$ substrate to a locally applied out-of-plane electric field (Fig. 4c) was calculated by introducing a pair of vertically separated point charges enclosing the molecule and $\mathrm{NaCl}$ cluster (charge of $+1.6 e$ and $-1.6 e$, with vertical position $z=-10 \AA$ and $+10 \AA$ with respect to the topmost $\mathrm{NaCl}$ layer, respectively, where $e$ is the unsigned elementary charge) at different lateral positions and relaxing the entire geometry.

\section{Charging probability calculation}

To calculate the spatial distribution of the LUMO charging probability (Fig. 2e, Extended Data Fig. 2b), we adopt the Bardeen model ${ }^{40}$, 


\section{Article}

where the tunnel current is proportional to the square of the matrix element between the relevant tip and sample wavefunctions. As in the Tersoff-Hamann approach ${ }^{40}$, we approximate the tip wavefunction to be spherical with an exponential decay length of $0.6 \AA\left(\right.$ ref. ${ }^{22}$ ), and we use the DFT-derived LUMO as the sample wavefunction. The best agreement between simulations and spatial maps of switching probability is obtained when the tip wavefunction is centred $9 \AA$ above the topmost $\mathrm{NaCl}$ layer.

\section{Data availability}

The data supporting the findings of this study are available from the corresponding authors upon request.

31. Alemani, M. et al. Electric field-induced isomerization of azobenzene by STM. J. Am Chem. Soc. 128, 14446-14447 (2006).

32. Zhang, Y. et al. Simultaneous and coordinated rotational switching of all molecular rotors in a network. Nat. Nanotechnol. 11, 706-712 (2016).

33. Stipe, B. C., Rezaei, M. A. \& Ho, W. Coupling of vibrational excitation to the rotational motion of a single adsorbed molecule. Phys. Rev. Lett. 81, 1263-1266 (1998).

34. Qiu, X. H., Nazin, G. V. \& Ho, W. Mechanisms of reversible conformational transitions in a single molecule. Phys. Rev. Lett. 93,196806 (2004).

35. Liljeroth, P., Repp, J. \& Meyer, G. Current-Induced hydrogen tautomerization and conductance switching of naphthalocyanine molecules. Science 317, 1203-1206 (2007).

36. Blum, V. et al. Ab initio molecular simulations with numeric atom-centered orbitals. Comput. Phys. Commun. 180, 2175-2196 (2009).
37. Perdew, J. P., Burke, K. \& Ernzerhof, M. Generalized gradient approximation made simple. Phys. Rev. Lett. 77, 3865 (1996)

38. Tkatchenko, A. \& Scheffler, M. Accurate molecular Van Der Waals interactions from ground-state electron density and free-atom reference data. Phys. Rev. Lett. 102, 073005 (2009).

39. Ruiz, V. G., Liu, W., Zojer, E., Scheffler, M. \& Tkatchenko, A. Density-functional theory with screened van der Waals interactions for the modeling of hybrid inorganic-organic systems. Phys. Rev. Lett. 108, 146103 (2012).

40. Chen, C. J. Introduction to Scanning Tunneling Microscopy (Oxford Univ. Press, 1993).

Acknowledgements We thank C. Meineke, A. Pöllmann, C. Rohrer, J. Hayes and M. Furthmeier for assistance and L. L. Patera for discussions. We acknowledge financial support from the Volkswagen Foundation (Lichtenberg programme) and Deutsche Forschungsgemeinschaft (DFG, German Research Foundation) through Project ID 422 314695032-SFB 1277 (Subproject B02) as well as Research Grant HU1598/3.

Author contributions D.P., L.Z.K., T.B., C.R., F.A., R.H. and J.R. conceived, set up and carried out the experiments. N.M. devised and carried out the DFT calculations. D.P., L.Z.K., T.B., C.R., R.H. and J.R. analysed the data and wrote the manuscript. All authors revised the manuscript.

Competing interests The authors declare no competing interests.

Additional information

Supplementary information is available for this paper at https://doi.org/10.1038/s41586-020 2620-2.

Correspondence and requests for materials should be addressed to R.H. or J.R.

Peer review information Nature thanks Nicholas Camillone and the other, anonymous,

reviewer(s) for their contribution to the peer review of this work.

Reprints and permissions information is available at http://www.nature.com/reprints. 


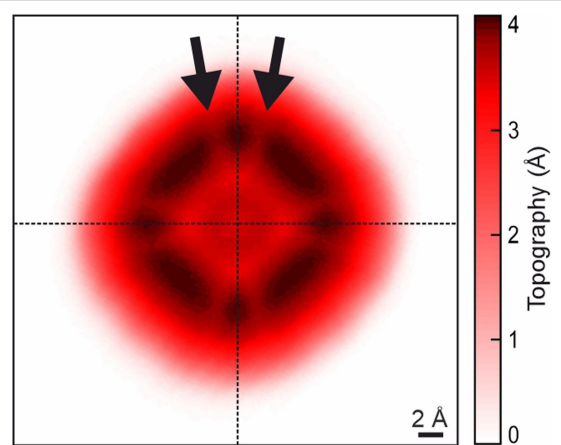

Extended Data Fig. 1 | Time-integrated STM image of recurringly switching MgPc molecule. Constant-current topographic map of the LUMO resonance of a MgPc molecule adsorbed on $\mathrm{NaCl}$ ( $1.1 \mathrm{~V}$ bias voltage, $2 \mathrm{pA}$ current setpoint). $\mathrm{The} \mathrm{NaCl}$ symmetry directions are indicated by dashed lines. A prominent ring of $4 \AA$ apparent height around the centre of the molecule is visible, featuring four pairs of local minima along the azimuthal direction (one pair indicated by arrows). To record each single pixel, millions of electrons are successively tunnelled from the tip into a molecular orbital and subsequently into the substrate, triggering the molecule to switch between both adsorption ground states $|l\rangle$ and $|r\rangle$ many times. As a result, the time-integrated LUMO density map features pairs of local minima deduced from the individual electronic nodal planes in states $|l\rangle$ and $|r\rangle$, representing a superposition of the electron density in both geometries. 


\section{Article}
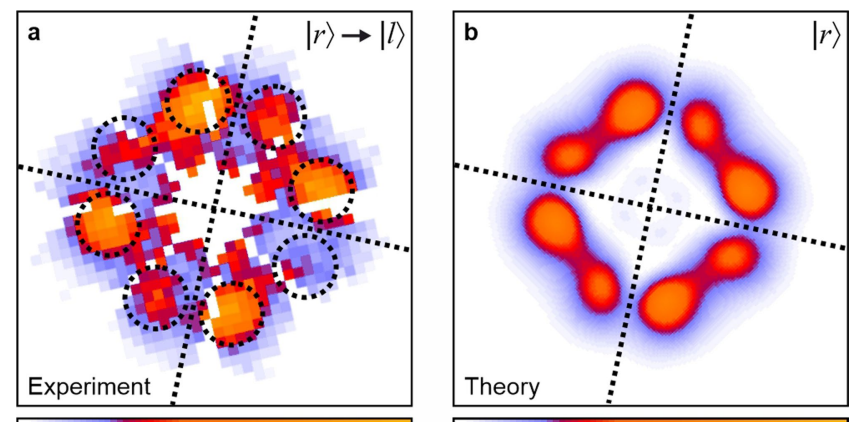

Switching probability 0.0475 $p_{\mathrm{r}-\mathrm{l}}$ (events/pulse)

0 Charging probability (a.u.) 1

Extended Data Fig. 2 | Path-selective map of the switching probability.

The panels show data for the same experiment and simulation as presented in

Fig. 2d, e, but here for the opposite switching direction from $|r\rangle$ to $|l\rangle$. 


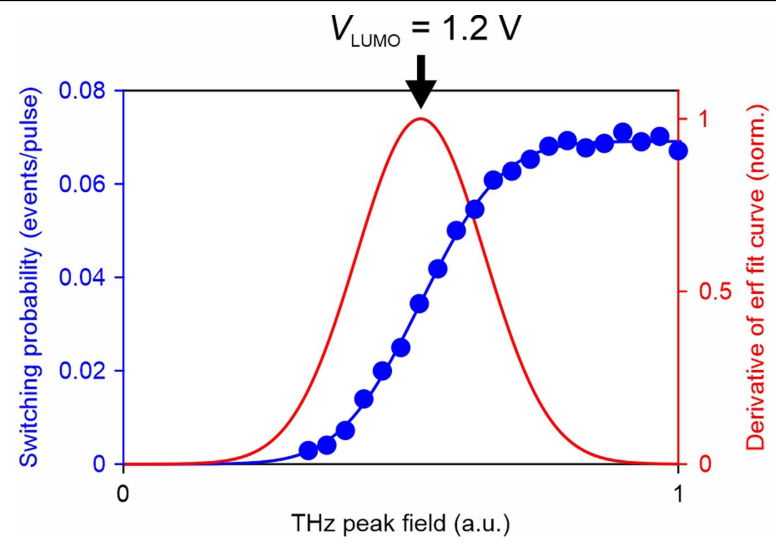

Extended Data Fig. 3 | Terahertz field calibration. The probability of switching triggered by individual terahertz pulses is measured as a function of terahertz field strengths (blue data points). The field strength is controlled by transmitting the pulses through a pair of crossed polarizers and plotted on a linear scale with initially undetermined scaling factor. As the field increases, the peak field of the pulses reaches the LUMO resonance. Assuming a linear relationship between the current resulting from tunnelling through the LUMO and the switching probability, the observed onset in switching probability with increasing field should roughly resemble the onset of the tunnelling current with increasing bias voltage in time-integrated scanning tunnelling spectroscopy. The Gaussian-shaped LUMO-related peak in time-integrated differential conductance $(\mathrm{d} / / \mathrm{d} V)$ spectra translates into an error-functionshaped onset of current with increasing voltage. Indeed, an error function (erf) fits the experimental data very well (blue fit curve), the derivative of which (red curve, normalized to the peak) reproduces the equivalent of a LUMO-related peak in $\mathrm{d} / \mathrm{d} V$ spectra. Time-integrated scanning tunnelling spectroscopy shows that the LUMO resonance is centred at a voltage of $1.2 \mathrm{~V}$, allowing for a calibration of the applied terahertz peak fields in terms of the transient's peak voltage. 
Article
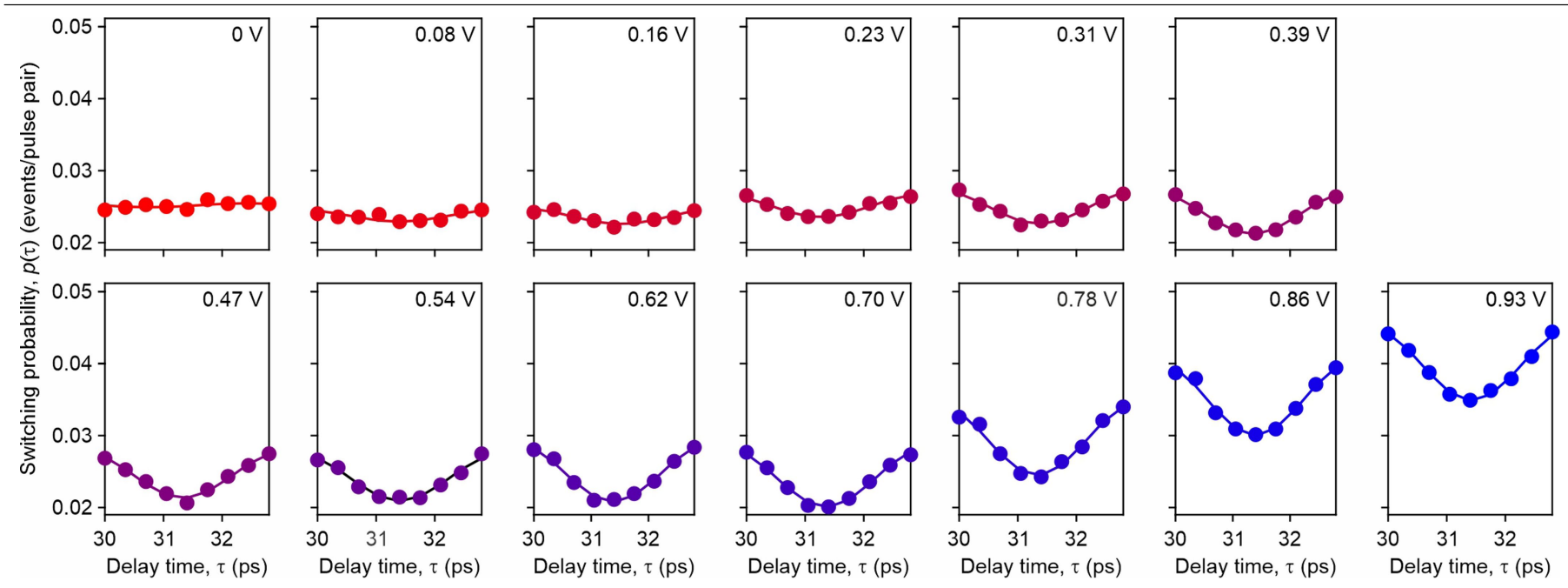

Extended Data Fig. 4 | Extraction of mean $\bar{p}$ and amplitude $p_{\mathrm{pp}}$ from coherently modulated switching probability traces. In a pump-probe scheme as depicted in Fig. Ba, pairs of terahertz pulses with an adjustable delay time $\tau$ induced switching events. Although the field strength of the probe pulses was chosen to facilitate electron tunnelling into the LUMO and thereby trigger switching events, the field strength of the pump pulses was systematically varied. For non-zero pump electric fields the time-resolved switching probability traces $p(\tau)$ show oscillations at a frequency of $0.3 \mathrm{THz}$, one period of which is resolved for different field strengths of the pump pulse. The latter is indicated in the top right corner of each panel, expressed as the peak voltage of the corresponding transient (calibrated via Extended Data Fig.3). For each curve, the time-averaged mean $\bar{p}$ and amplitude $p_{\mathrm{pp}}$ are extracted by fitting a sine (solid lines) to the data. 


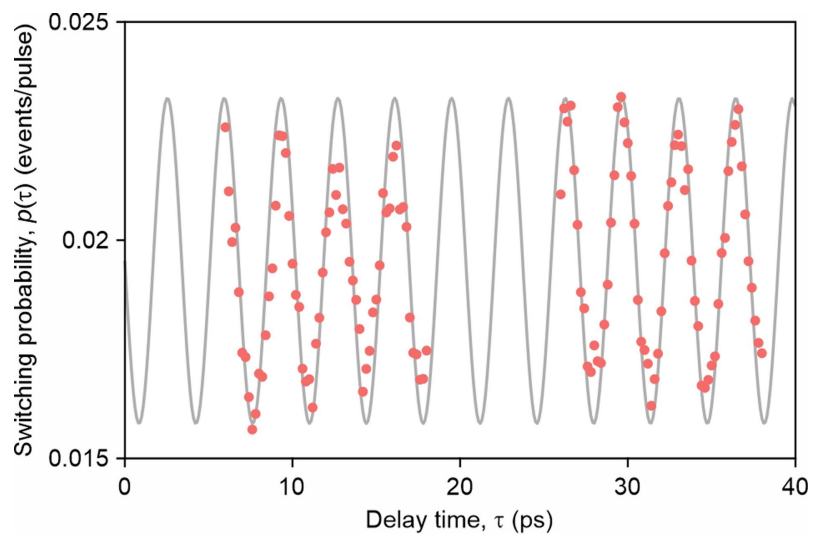

Extended Data Fig. 5 | Field-induced coherence modulates switching

probability by $39 \%$. Time-resolved switching probability versus delay time, obtained in the same way as the data shown in Fig. 3b. Here, the field strength of pump and probe pulses were equal and individually large enough to facilitate tunnelling into the LUMO, which corresponds to a peak value of the corresponding voltage transient of about $1.2 \mathrm{~V}$; see Extended Data Fig. 3. This data exhibits a field-induced modulation of $39 \%$, representing the largest variation that we detected (peak-to-peak modulation of 0.0076 to an average of 0.0195). 
a

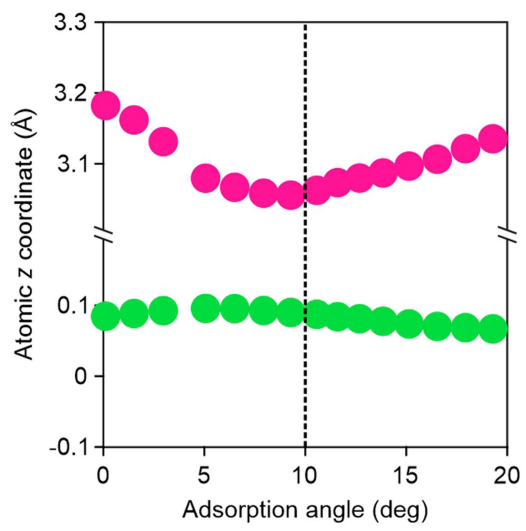

b

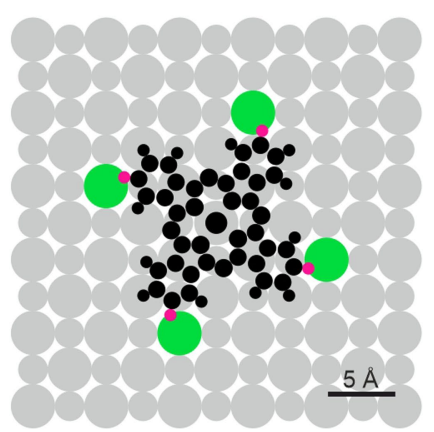

c

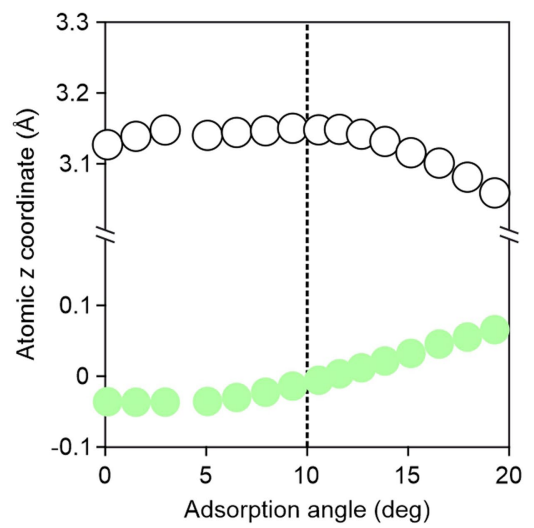

d

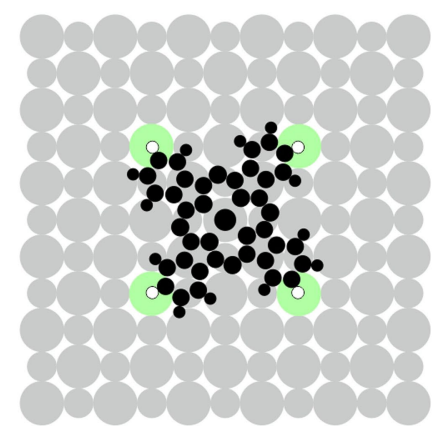

e

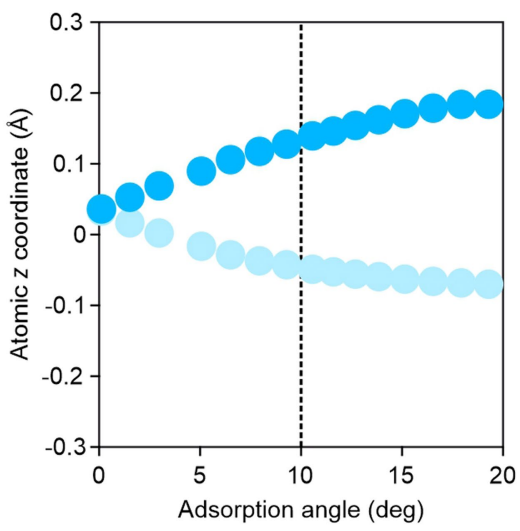

f

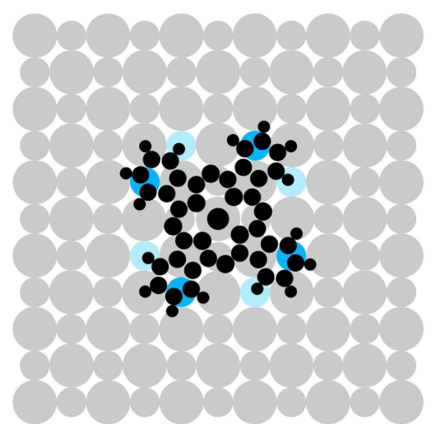

Extended Data Fig. 6 | Analysis of the role of specific atoms of MgPc and the $\mathrm{NaCl}$ substrate in determining the adsorption energetics with respect to the azimuthal orientation. In DFT calculations we enforce different in-plane rotation angles by fixing one of the in-plane coordinates of two nitrogen atoms of the molecule and the orientation of the substrate while relaxing the remaining geometry. Performing this calculation for several angles allows us to track the response of select atoms to an in-plane rotation of MgPc. a, Pink data points indicate the $z$ coordinate (perpendicular to the surface) of four equivalent hydrogen atoms of the molecule (highlighted in pink in $\mathbf{b}$, top view of molecule and $\mathrm{NaCl}$ layers) as a function of adsorption angle and show a clear excursion of the atoms towards the substrate for rotation angles around $\phi=10^{\circ}$ ( $z$ coordinates relative to the topmost $\mathrm{NaCl}$ layer). Similarly, the $z$ coordinate of the closest $\mathrm{Cl}^{-}$ion (green data points in $\mathbf{a}$ and green atoms in $\mathbf{b}$ ) shows a clear upward deflection at similar angles, suggesting a local interaction between these two atoms. c, d, For comparison, we show the $z$ coordinates of other pairs of $\mathrm{H}$ atoms and $\mathrm{Cl}^{-}$ions, which do not exhibit such behaviour. e, f, Further, the $z$ coordinate of four equivalent sodium atoms (intense blue) located below one of the $\mathrm{C}-\mathrm{C}$ bonds of the molecule's peripheral benzene rings indicates an excursion towards the molecule of up to almost 0.2 A for increasing adsorption angles. For comparison, we plot the $z$ coordinates of another sodium atom (light blue), which has an analogous role in the opposing switch position. This atom exhibits an excursion away from the molecule. Notably, all three atoms that feature a strong coupling between their $z$ coordinate and the in-plane rotation of the molecule (pink, intense green, intense blue) are located in the region where local fields induce the strongest coherent excitation (Fig. 4). 